\title{
Modelling and Reporting Parameters of Optical OFDM System Using Different Modulation Techniques
}

\author{
Harsimran Jit Kaur ${ }^{1,2}$, Maninder Lal Singh ${ }^{1,2}$ \\ ${ }^{1}$ Electronics and Communication Engineering, Chitkara University, Mohali, India \\ ${ }^{2}$ Guru Nanak Dev University, Amritsar, India \\ Email: Harsimran.kaur@chitkara.edu.in,mlsingh7@gmail.com
}

Received December 5, 2012; revised April 1, 2013; accepted April 10, 2013

Copyright (C) 2013 Harsimran Jit Kaur, Maninder Lal Singh. This is an open access article distributed under the Creative Commons Attribution License, which permits unrestricted use, distribution, and reproduction in any medium, provided the original work is properly cited.

\begin{abstract}
OFDM (Optical Orthogonal Frequency Division Multiplexing) in the optical domain is a promising technology which provides solutions to problems of today's optical networks. In the present research an optical OFDM system is designed using different modulation techniques QPSK (quadrature phase shift keying), QAM (quadrature amplitude Modulation). Various signal conditioning parameters, like OSNR (optical signal to noise ratio), BER (bit error rate), chromatic dispersion and electrical SNR (signal to noise ratio) have been reported in present research. These parameters could be used for identifying the fault. On the basis of conclusion drawn for values of these parameters, the system may dynamically adapt and reconfigure itself for better transmission. In present research OPTSIM and MATLAB has been used as Simulation tools.
\end{abstract}

Keywords: OFDM; OSNR; BER; QPSK

\section{Introduction}

Recently, Orthogonal Frequency Division Multiplexing (OFDM) is emerging as leading technology for high data rates. It has evolved to be leading communication standard in almost every major standard (Wi-Max, IEEE 802.11a, DVB) [1,2]. OFDM is based on Multi carrier transmission which transforms a wide-band channel into a group of narrowband channels [3]. OFDM offers two fundamental advantages. One is its robustness against channel dispersion and second is ease of phase and channel estimation in a time-varying environment. OFDM is benefited from the fact that it is using digital signal processing of fast-Fourier transforms (FFTs) to obtain high sub-carrier density and computationally-efficient phase and amplitude equalization [4].

Even though OFDM technology is having a number of important advantages and is widely accepted standard in wireless communications, but it is only very few years back that it has been considered for optical communications [5]. This was because few years back the silicon signal processing technology was not capable of dealing with sophisticated OFDM signal processing in a CMOS integrated circuit. It is also very recent that demand for such high data rates for long distances has occurred.
It has been proposed that the concept of software-defined radio (SDR) is a practical solution, for software implementation of the radio transceiver which is capable of adapting itself dynamically to the user environment instead of relying on dedicated hardware [6,7]. There should be software-defined optical transmission in which the system is capable of reconfiguring itself for various parameters [1]. This system should be capable of various signal conditioning parameters, like OSNR, chromatic dispersion, and electrical Signal to Noise Ratio (SNR). We can use these parameters for identifying the fault. On the basis of conclusion drawn for values of these parameters system may dynamically adapts and reconfigure itself for better transmission.

In the present research a system is demonstrated by using the simulative environment for optical fiber in OPTSIM software, which allows the design of many configurations regarding optical communications. In this work, an optical OFDM transmission is simulated. The programming of the OFDM coder and decoder has been done with MATLAB software.

\section{Optical OFDM System}

OFDM transmits information on high speed data channel 
by dividing it into blocks of data. An optical OFDM transmitter could be altered for different fiber types, different network ranges varying from short to long-haul for different types of detection including direct or coherent detection. System would be designed for providing bitrate flexibility by using different modulation depth. A block diagram of optical OFDM transmitter diagram is shown in Figure 1. The system uses Fourier transform techniques to encode the data in which the data information is carried over many lower rate subcarriers [8]. System transmits OFDM modulated data over optical channel. Transmission system is divided into three parts; transmitter, channel, receiver. Transmitter section involves presenting data into $\mathrm{N}$ parallel paths where data at each path could be modulated using various digital modulation techniques including QPSK (Quadrature phase shift keying), 16QPSK, QAM (Quadrature amplitude modulation) etc. Basic requirement of OFDM technique for multiple microwave mixers producing various sub-carriers frequencies could be fulfilled by using an inverse-FFT (IFFT) for generating dense comb of OFDM sub-carrier frequencies [9]. Serial to parallel and parallel to serial conversions are performed for converting random generated data with high rate into low data rate and vice versa.

\section{Performance Monitoring Parameters}

Performance monitoring is very important feature for optical systems. Though there exists number of criteria for monitoring performance of optical transmission systems but their correct choice is an important issue for an effective design of future long-haul optical systems. Various important parameters which are reported through present research include BER (Bit error rate), OSNR (Optical signal to noise ratio), jitter, eye opening, distortion, dispersion etc.

Performance of channel could be directly determined by the BER, which is depending upon OSNR, dispersion and non linear effects. OSNR is very important and dominant performance parameter which could help in link optimization by limiting dispersion and non linearity $[10,11]$.

\section{Simulation Model}

In the present research the programming of the OFDM coder and decoder has been done with MATLAB software. For monitoring and analysing various parameters, the optical OFDM transmission system is modelled using OPTSIM. OFDM modulated data is interfaced with OPTSIM to carry the transmission over fiber.

In the generation of OFDM signal binary data from random generator is obtained. Interleaving is performed on these binary data to spread the errors out in the bitstream. These binary data values are mapped to symbols, using QPSK or one of the M-ary QAM techniques. A training frame of pilot sub-carriers of length 96 samples is inserted which is sent prior to sending information. This could be used at receiver to carry out channel estimation for compensating the effect of channel on transmitted signal. To generate OFDM symbol data containing complex words frame and pilots frame are passed through 64-bit IFFT. The frame is extended by insertion of zeros to reduce the effect of Inter-carrier Interference. Cyclic prefix of 16 bits is added which consists of the end of the OFDM symbol to be copied into the guard interval, and the guard interval is transmitted followed by the OFDM symbol. This would help the receiver to integrate over an integer number of sinusoid cycles during performing OFDM demodulation with the FFT.

OFDM data is then converted to serial from parallel by using Parallel-to-Serial $(\mathrm{P} / \mathrm{S})$ converter. OFDM modulated data is interfaced with OPTSIM to carry over optical transmission.

Simulation model of an optical communication system at $10 \mathrm{~Gb} / \mathrm{s}$ around $1550 \mathrm{~nm}$ central wavelength is shown in Figure 2. The simulation is using OPTSIM to carry transmission over fiber. Simulation is done for 100 $\mathrm{km}$ length of standard single-mode (SM) fiber. Standard $\mathrm{SM}$ fiber has attenuation of $0.2 \mathrm{~dB} / \mathrm{km}$, and dispersion 16

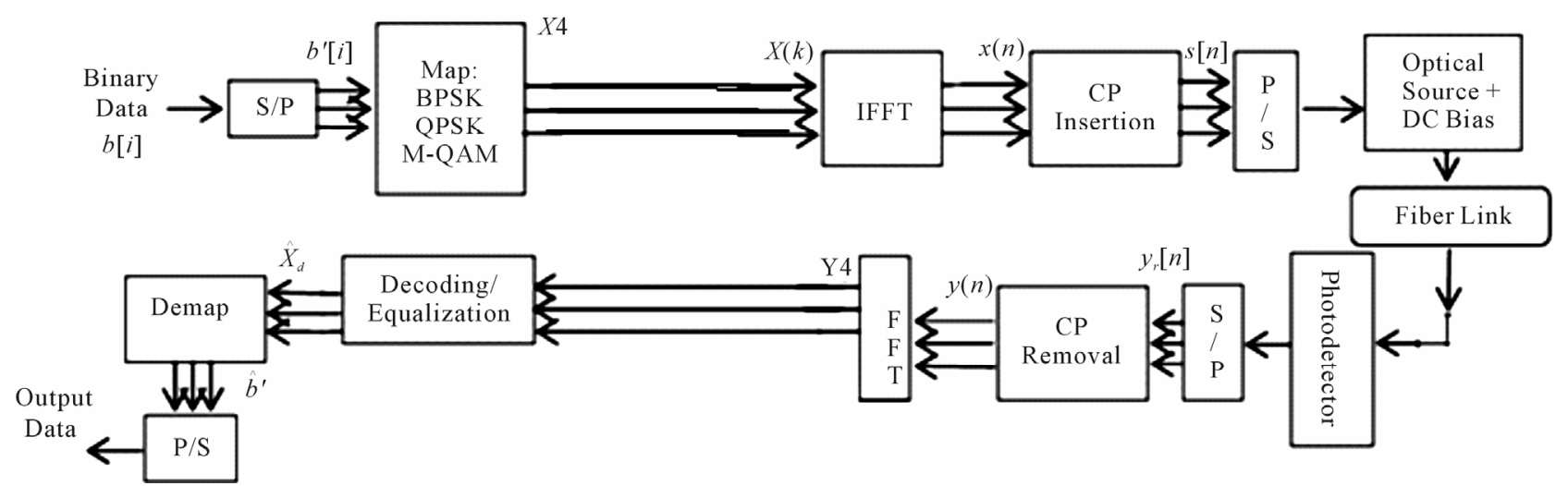

Figure 1. Optical OFDM system block diagram. 


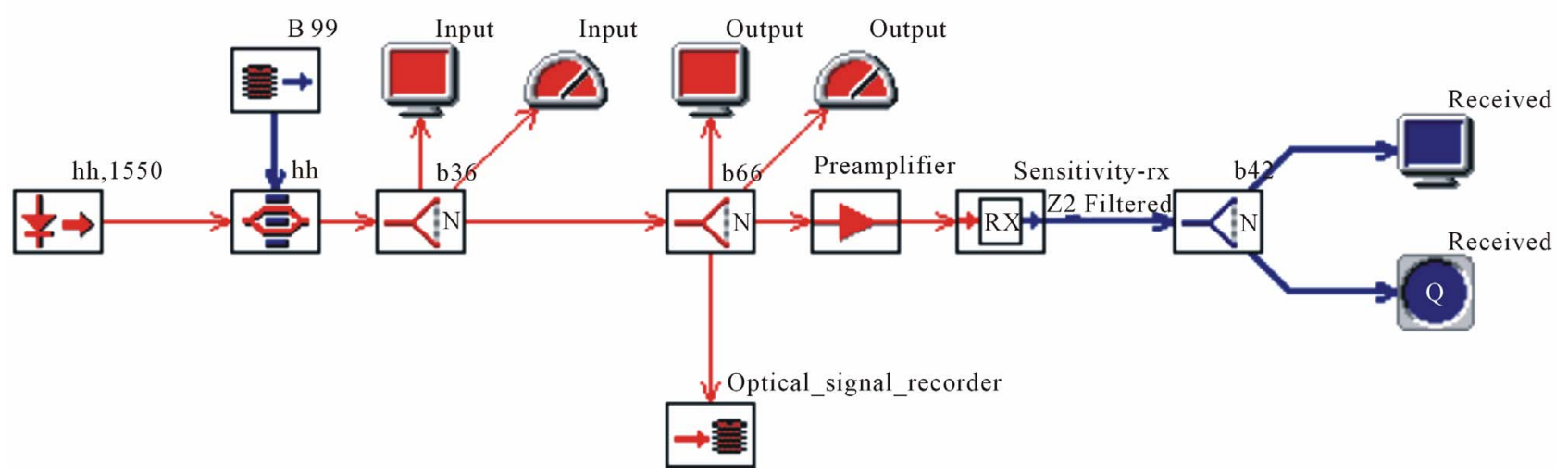

Figure 2. System model for optical transmission.

$\mathrm{ps} / \mathrm{nm} / \mathrm{km}$ at reference frequency. It has zero dispersion at $1391.53354633 \mathrm{~nm}$ wavelength, fiber average beat length $5 \mathrm{~m}$. CW Lorentzian Laser used was having center emission wavelength $1550 \mathrm{~nm}, \mathrm{CW}$ power $1 \mathrm{~mW}$ and FWHM linewidth $10 \mathrm{MHz}$ as main characteristics was used as the optical source. Amplitude dual-arm Mach Zehnder modulator is used here to modulate the optical signal of desired format having the following parameters: excess loss $0 \mathrm{~dB}$, offset voltage corresponding to the phase retardation in the absence of any (on both arms) electric field $0.5 \mathrm{~V}$, extinction ratio $20 \mathrm{~dB}$, chirp factor 0 and average power reduction due to modulation $3 \mathrm{~dB}$. At the receiver section reverse operations are employed by doing serial to parallel conversion, removing cyclic prefix. FFT operations are performed to recover sub-carriers and finally de-mapping of M-ary QAM, QPSK is done to binary values.

\section{Simulation Results}

This section is reporting simulation results for various parameters of optical OFDM system. Various important parameters values used during simulation are listed in Table 1.

Figure 3 is depicting the eye diagram for the simulated system. In the present system the transmitter generates QPSK modulated OFDM signal which passes through transmission link SMF and at receiver demodulated back to carry over various evaluations. Table 2 represents set of certain evaluation parameters obtain for this simulated system.

In the present research QAM is other modulation technique which is selected for transmitting signal through fiber link. Figure 4 is representing the eye diagram for QAM modulated optical OFDM system. It is representing quite good eye opening of $0.150 \mathrm{e}-06$ with very small BER of $6.564 \mathrm{e}-013$.

Evaluation parameters for QAM modulated system is tabulated in Table 3.

On comparing the results of BER of QPSK and QAM
Table 1. Optical OFDM simulation parameters.

\begin{tabular}{cc}
\hline Parameter & Value \\
\hline Data rate & $10 \mathrm{Gbps}$ \\
Wavelength & $1550 \mathrm{~nm}$ \\
Fiber length & $100 \mathrm{~km}$ \\
CW laser frequency & $193.1 \mathrm{THz}$ \\
Gain & $35 \mathrm{~dB}$ \\
Cyclic prefix & $16 \mathrm{bit}$ \\
FFT & $64 \mathrm{bits}$ \\
\hline
\end{tabular}

Table 2. Simulation results for QPSK modulated system.

\begin{tabular}{cc}
\hline Parameter & Value \\
\hline BER & $7.06425 \mathrm{e}-012$ \\
Eye opening & $0.0723 \mathrm{e}-006$ \\
Jitter & 0.0201543 \\
Q factor & $19.98 \mathrm{~dB}$ \\
\hline
\end{tabular}

Table 3. Simulation results for QAM modulated system.

\begin{tabular}{cc}
\hline Parameter & Value \\
\hline BER & $6.5624 \mathrm{e}-013$ \\
Eye opening & $0.158919 \mathrm{e}-06$ \\
Jitter & $22.24 \mathrm{~dB}$ \\
Q factor & $18.99 \mathrm{~dB}$ \\
\hline
\end{tabular}

modulated systems we can find out that BER for QAM modulated system is better than QPSK system. The eye diagram of QPSK is showing closer then QAM system.

OSNR is one very important parameter for estimating the quality of transmission. It is defined as ratio of signal power and noise power. We can increase OSNR by increasing the output optical power. Among the various 


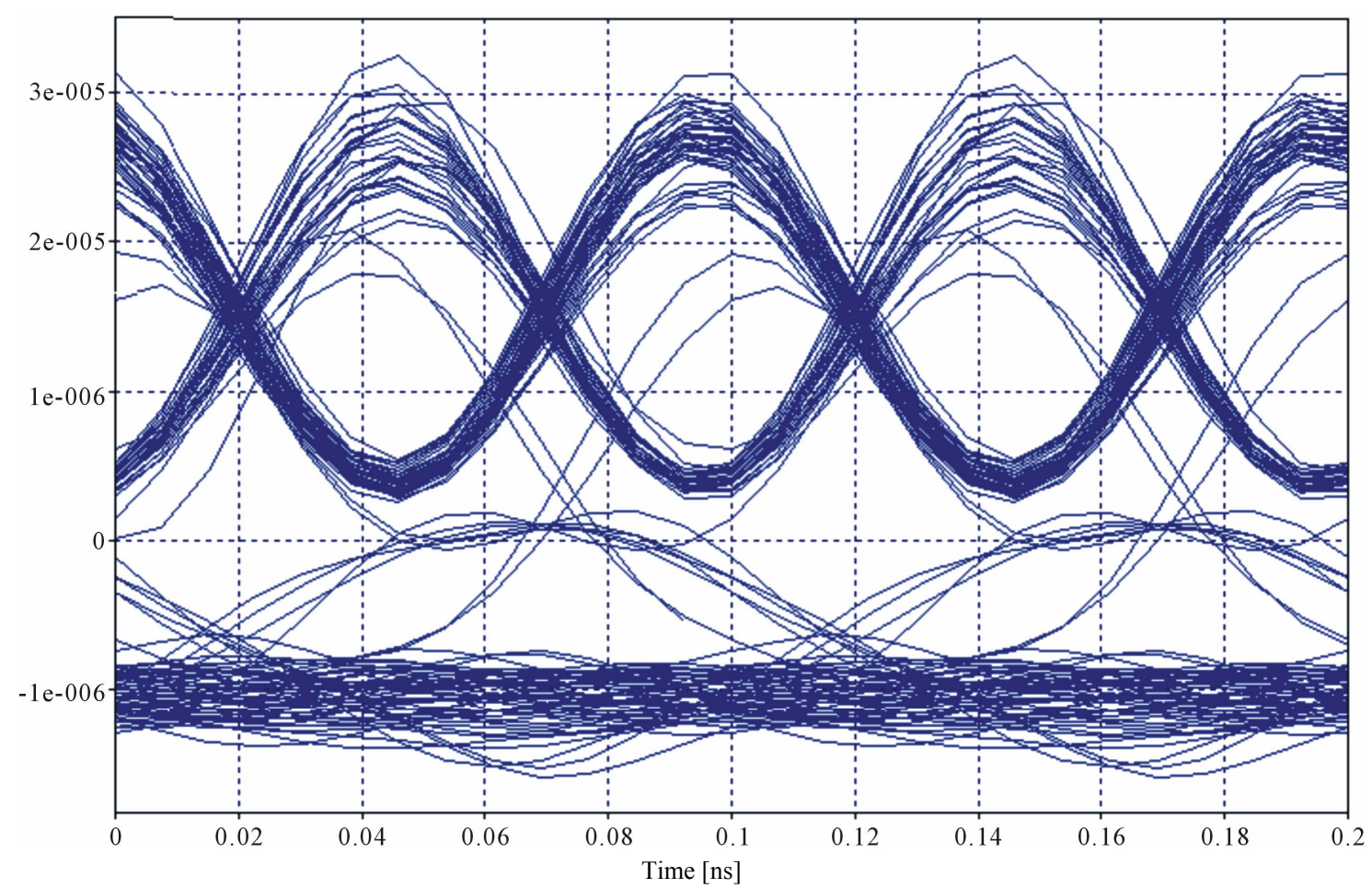

Figure 3. Eye diagram for QPSK transmission.

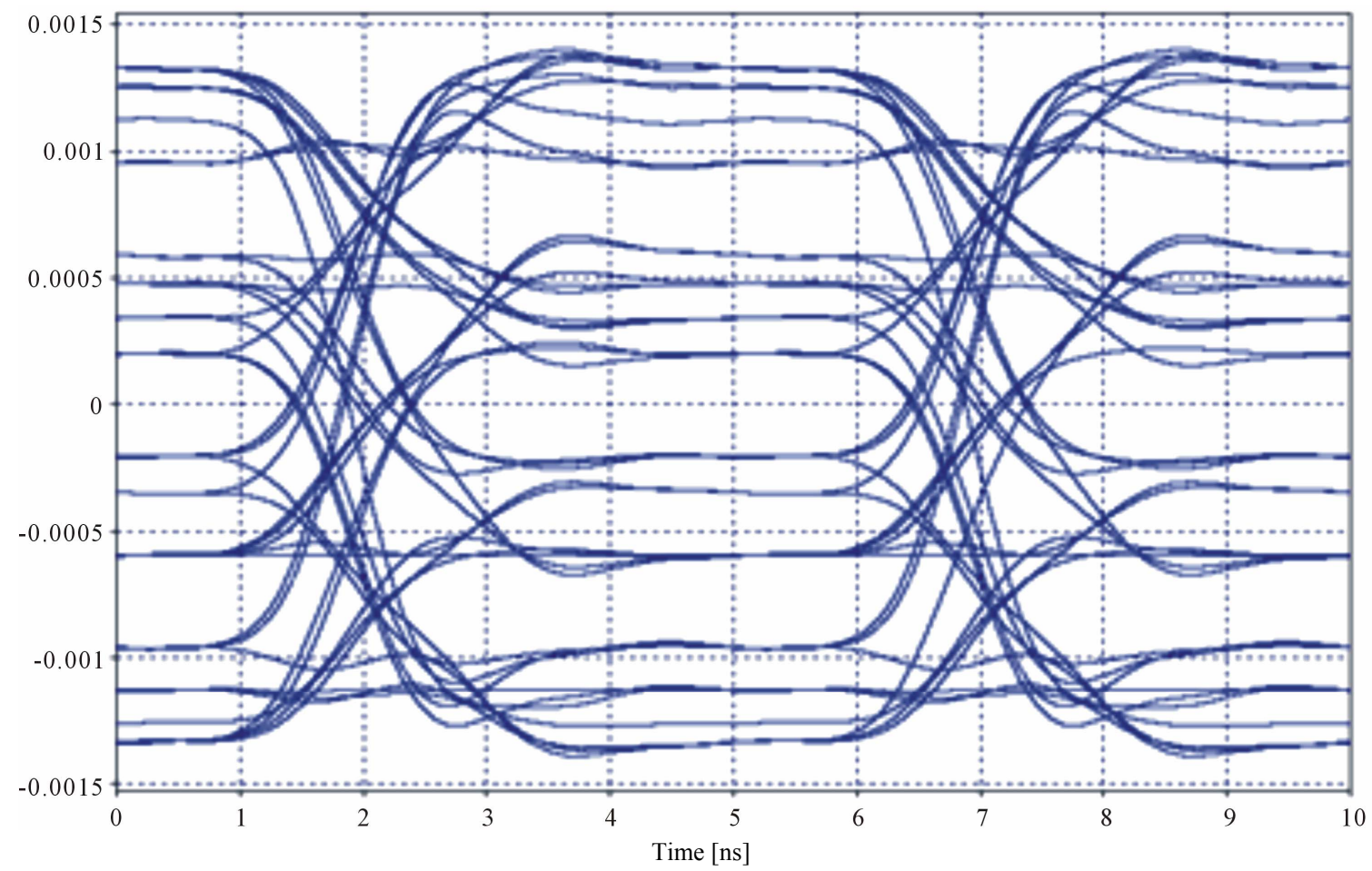

Figure 4. Eye diagram for QAM Transmission.

analog performances monitoring techniques of optical spectrum analysis OSNR measurement is very common.

It has been stated that to achieve a minimum BER of $\mathrm{e}^{-}-03$ OSNR of $11 \mathrm{~dB} / 0.1 \mathrm{~nm}$ is required [1]. Figure 5 is showing the BER performance for optical OFDM transmission over $100 \mathrm{~km}$. OSNR value for BER e-012 is $17.7 \mathrm{~dB} / 0.1 \mathrm{~nm}$. It could be clearly concluded from Figure 5 that OSNR value required for BER e-03 is 11.9 
$\mathrm{dB} / 0.1 \mathrm{~nm}$.

For channel estimation among the various performances monitoring parameters includes, chromatic dispersion. The cause of occurrence of chromatic dispersion is difference in group velocities among the different spectral components. In the present research chromatic dispersion has been analyzed with reference to wavelength. Figure 6 is depicting the curve which shows the chromatic dispersion change as function of frequencies.

It has been stated that chromatic dispersion varies in the range $15-18 \mathrm{ps} /(\mathrm{km}-\mathrm{nm})$ near $1.5 \mu \mathrm{m}-1.55 \mu \mathrm{m}$ [12]. The fiber loss is minimum near $1.55 \mu \mathrm{m}$ so this region is of significant interest [12]. From Figure 6 it is clearly concluded that in the present simulated system dispersion is varying between $12-17 \mathrm{ps} /(\mathrm{km}-\mathrm{nm})$ near $1.5 \mu \mathrm{m}$ $1.55 \mu \mathrm{m}$. Present simulated system is giving quite smaller dispersion in range $1.5 \mu \mathrm{m}-1.55 \mu \mathrm{m}$.

Table 4 is summarizing Chromatic dispersion (ps/ $\mathrm{km}-\mathrm{nm}$ ) at different wavelengths.

It is very important to analyze the pulse broadening

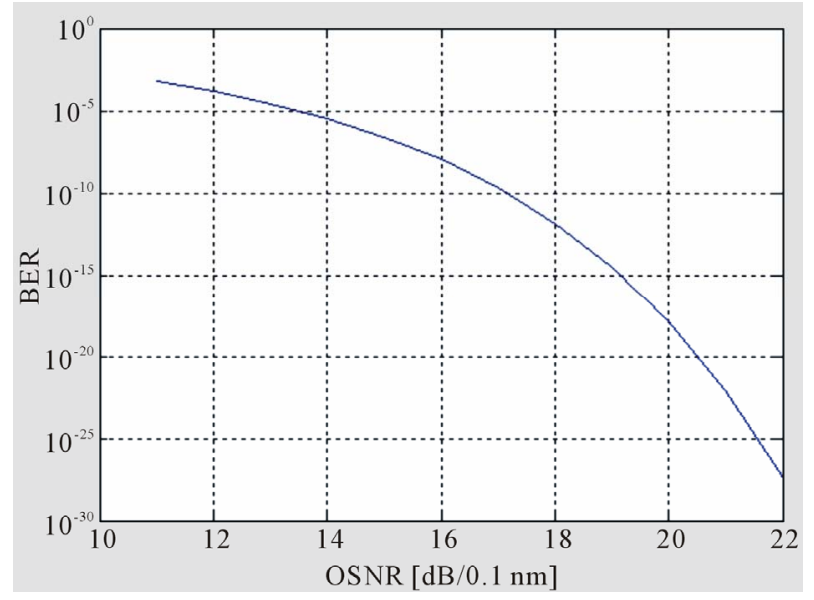

Figure 5. BER performance vs OSNR of optical OFDM system.

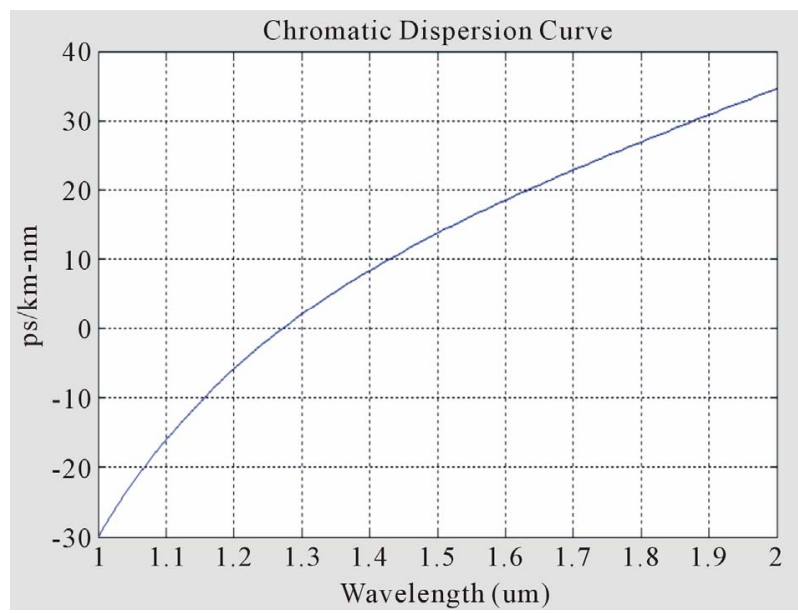

Figure 6. Chromatic dispersion versus wavelength. produced with distance. In the present research the effect of dispersion for different chirp factors has been analyzed. Figure 7 is showing the performance analysis curve for comparing pulse broadening produced for different chip factors. From the Figure $\mathbf{7}$ it is clear that the broadening increases linearly for positive and negative values of chirp factor. The broadening is minimum when chirp factor is zero.

SNR is another very important figure of merit for monitoring the performance of transmission quality. Figure 8 is showing performance comparison curve for BER and signal to noise ratio for QAM optical OFDM system. This curve is comparing the performance for theoretical and present optical OFDM system. Simulation is conducted for a QAM modulated OFDM system and compare the numerical simulation result with that obtained theoretically using the analytical expression of Equation (1) [13].

$$
\mathrm{BER}=1-\left(1-\frac{1}{\log _{2} \mathrm{M}}\left(1-\frac{1}{\sqrt{\mathrm{M}}}\right) \mathrm{Q}\left(\sqrt{\frac{3}{\mathrm{M}-1} \frac{\mathrm{S}}{\mathrm{N}_{0}}}\right)\right)
$$

$\frac{\mathrm{S}}{\mathrm{N}_{0}}$ is signal to noise ratio, $\mathrm{M}$ is number of constellation points, BER is bit error rate.

The result is shown in Figure 8. It is observed from this that the theoretical BER predicts a gain of almost

Table 4. Chromatic dispersion versus wavelength.

\begin{tabular}{cc}
\hline Wavelength $(\mathrm{nm})$ & Chromatic dispersion $(\mathrm{ps} / \mathrm{km}-\mathrm{nm})$ \\
\hline 1.3 & 7 \\
1.5 & 17 \\
1.7 & 21 \\
1.8 & 25 \\
\hline
\end{tabular}

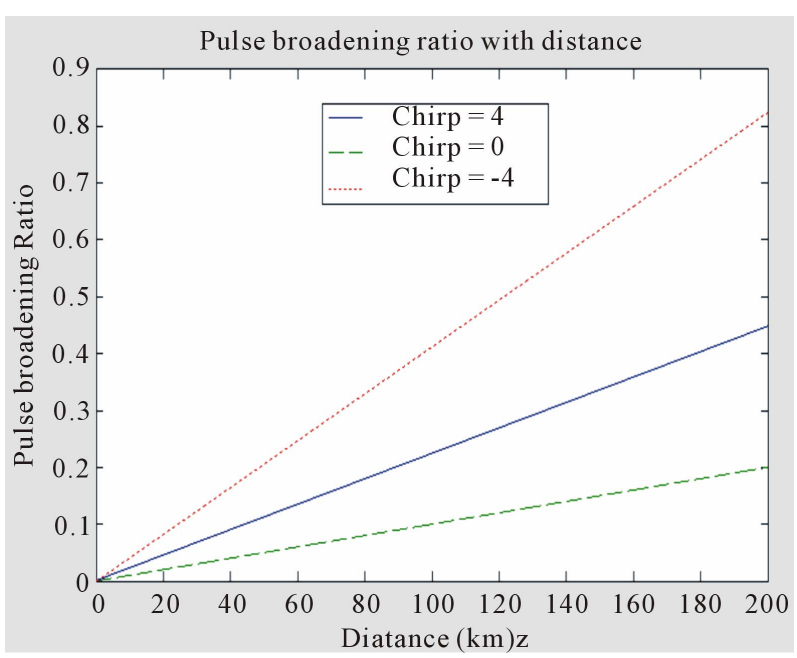

Figure 7. Pulse broadening ratio with distance. 


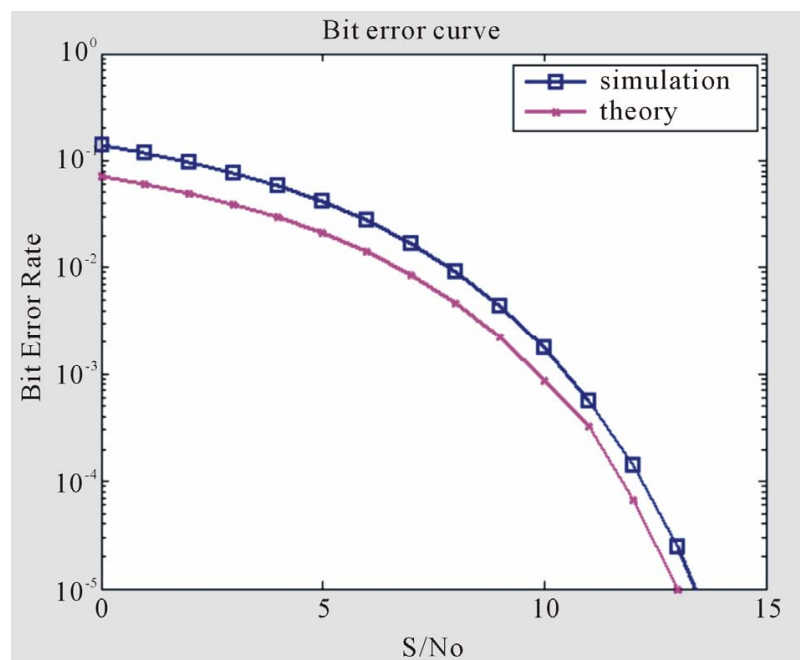

Figure 8. BER performance of QAM system both the results of numerical simulation and analytical calculation are shown.

$11.5 \mathrm{~dB}$ at a BER of $10^{-4}$. Note that the experimental results suggest a gain of about $13 \mathrm{~dB}$ at same BER, which is not far from the theoretical prediction, but may suggest additional noises and losses in fiber channel during transmission. Simulated Optical OFDM system is performing $1.5-2 \mathrm{~dB}$ worse than the theoretical formula. Theoretical formulas do not consider synchronization errors and quantization of received samples which degrade the performance of system [14].

\section{Validation of Simulated Results}

It has been proposed that OFDM is a best suited technique for high speed applications as in this method, transmission of data blocks is done using parallel processing. This technique uses FFT which is implemented very efficiently using digital signal processors [1]. Q factor $16.9 \mathrm{~dB}$ corresponds to BER value of $10 \mathrm{e}^{-12}$, whereas $\mathrm{Q}$ factor 18.06 $\mathrm{dB}$ correspond to BER value of $10 \mathrm{e}^{-15}$ [1]. This simulation test bed designed for optical OFDM transmission is reporting $\mathrm{Q}$ factor of $18.99 \mathrm{~dB}$ with BER value $6.5624 \mathrm{e}^{-}$ 013 at eye opening of $0.158919 \mathrm{e}-06$ for QAM modulated system whereas for QPSK modulated system Q factor values are $19.98 \mathrm{~dB}$ with BER 7.06425e-012 at eye opening of $0.0723 \mathrm{e}^{-006}$. The simulation results for BER performance for optical OFDM system has reported BER value e- 05 at OSNR of $13 \mathrm{~dB}$ [1]. Present simulated system is reporting BER e-05 at OSNR $13.9 \mathrm{~dB} / 0.1 \mathrm{~nm}$.

Typical values of dispersion varies in the range $15-18$ $\mathrm{ps} /(\mathrm{km}-\mathrm{nm})$ near $1.5 \mu \mathrm{m}-1.55 \mu \mathrm{m}$ [12]. The fiber loss is minimum near $1.55 \mu \mathrm{m}$ so this region is of significant interest [12]. In the present simulated system dispersion is varying between 12 - $17 \mathrm{ps} /(\mathrm{km}-\mathrm{nm})$ near $1.5 \mu \mathrm{m}-1.55$ $\mu \mathrm{m}$. It has been reported that the broadening increases linearly for positive value of chirp factor. Minimum broad- ening is obtained when chirp factor is zero [15].

\section{Conclusions}

Modelling and comprehensive analysis of optical OFDM transmission has been carried out in the present research. The main conclusions drawn during the present research are that the BER value of the QAM modulator is better than QPSK modulator. QPSK OFDM transmission shows good eye opening of $0.0723 \mathrm{e}-006$ with BER of $7.06425 \mathrm{e}-012$.

BER performance with OSNR has been presented which reports OSNR value of $13.9 \mathrm{~dB} / 0.1 \mathrm{~nm}$ for BER of $\mathrm{e}-005$. It has been observed that dispersion is varying between 12 - $17 \mathrm{ps} /(\mathrm{km}-\mathrm{nm})$ near $1.5 \mu \mathrm{m}-1.55 \mu \mathrm{m}$. On comparison of the performance for theoretical and present optical OFDM system it is observed that simulated Optical OFDM system is performing $1.5-2 \mathrm{~dB}$ worse than the theoretical formula. The reason for this difference is that theoretical formulas do not consider synchronization errors and quantization of received samples which degrade the performance of the system.

\section{REFERENCES}

[1] W. Shieh and I. Djordjevic, "Orthogonal Frequency Division Multiplexing for Optical Communication," Academic Press, Waltham, 2010.

[2] J. G. Proakis and M. Salehi, "Fundamentals of Communications Systems Engineering," 3rd Edition, Prentice Hall, Upper Saddle River, 2005.

[3] V. J. Amirtha Vijina and J. Jayalakshmi, "Modelling and Performance Analysis of Optical OFDM System Using 16-QAM and 64-QAM Modulation Technique Using Simulink Tool," International Journal of Systems, Algorithms \& Applications, Vol. 2, No. 5, 2012, pp. 9-12.

[4] A. Lowery and J. Armstrong, "Orthogonal-FrequencyDivision Multiplexing for Dispersion Compensation of Long-Haul Optical Systems," Optics Express, Vol. 14, No. 6, 2006, pp. 2079-2084. doi:10.1364/OE.14.002079

[5] J. Armstrong, "OFDM for Optical Communications," Journal of Lightwave Technology, Vol. 27, No. 3, 2009, pp. 189-204.

[6] A. Abidi, "The Path to the Software-Defined Radio Receiver," IEEE Journal of Solid-State Circuits, Vol. 42, No. 5, 2007, pp. 954-966.

[7] J. Mitola, "The Software Radio Architecture," IEEE Communications Magazine, Vol. 33, No. 5, 1995, pp. 26-38. doi:10.1109/35.393001

[8] R. Hui, B. Zhu, R. Huang, C. T. Allen, K. R. Demarest and D. Richards, "Subcarrier Multiplexing for High Speed Optical Transmission," Journal of Lightwave Technology, Vol. 20, No. 3, 2002, pp. 417-427. doi: $10.1109 / 50.988990$

[9] S. Hara and R. Prasad, "Multicarrier Techniques for 4G Mobile Communications," Artech House, Boston, 2003.

[10] F. Forghieri, R. W. Tkach and D. L. Favin, "Simple Mo- 
del of Optical Amplifier Chains to Evaluate Penalties in WDM Systems," IEEE/OSA Journal of Lightwave Technology, Vol. 16, No. 9, 1998, pp. 1570-1576. doi: $10.1109 / 50.712238$

[11] R. S. Fyath and A. A. Al-Mfrji, "Performance Evaluation of Multimode Fiber-Based Optical OFDM Communication System," Proceedings of 1st Regional Conference of Engineering Sciences of Nahrain University, College of Engineering, Vol. 11, No. 1, 2008, pp. 70-83.

[12] G. P. Agrawal, "Fiber-Optic Communication Systems," 2nd Edition, Academic Press, Waltham, 2002. doi:10.1002/0471221147

[13] R. Noe, "Essentials of Modern Optical Fiber Communi- cations," Springer, Berlin, 2010. doi:10.1007/978-3-642-04872-2

[14] W. C. Cox, J. A. Simpson, C. P. Domizioli, J. F. Muth and B. L. Hughes, "An Underwater Optical Communication System Implementing Reed-Solomon Channel Coding," Photonics Technology Letters, Vol. 1, No. 3, 2008, p. 978.

[15] Md. Jahidul Islam, Md. Saiful Islam and Md. Mahmudur Rahman, "Dispersion Compensation in Optical Fiber Communication Using Fiber Bragg Grating," Global Journal of Researches in Engineering, Vol. 12, No. 2, 2012, pp. 24-29. 